\title{
Avaliação da qualidade dos sedimentos em áreas agrícolas do município de Bom Retiro/SC
}

O município de Bom Retiro/SC se caracteriza por intensa atividade agropecuária. Essas atividades podem impactar os recursos naturais, devido a utilização de agrotóxicos e fertilizantes que podem conter metais, causando diversos impactos ambientais. Com este estudo, objetivou-se caracterizar os sedimentos das áreas rurais influenciadas por atividades agropecuárias no município de Bom Retiro/SC. Para representação significativa da área, foram realizadas coletas de sedimentos em 25 pontos influenciados diretamente pelas referidas atividades. Os metais pesados cádmio $(\mathrm{Cd})$, cobre $(\mathrm{Cu})$, $\mathrm{cromo}(\mathrm{Cr})$, ferro ( $\mathrm{Fe})$, níquel (Ni), chumbo ( $\mathrm{Pb})$ e zinco $(\mathrm{Zn})$ foram determinados por Espectrometria de Absorção Atômica de Alta Resolução com Fonte Contínua (ARFC EAA). Os sedimentos superficiais apresentaram concentrações de $\mathrm{Cu}, \mathrm{Cr}, \mathrm{Fe}, \mathrm{Ni}, \mathrm{Pb}$ e Zn, mas todos os pontos estavam enquadrados pela Resolução CONAMA $\mathrm{n}^{\circ} 454 / 2012$ como nível 1.

Palavras-chave: Qualidade dos sedimentos; Metais pesados; Produção agrícola.

\section{Sediment quality assessment in agricultural areas in the Bom Retiro/SC}

\begin{abstract}
The municipality of Bom Retiro/SC is characterized by intense agricultural activity. These activities can impact natural resources, due to the use of pesticides and fertilizers that may contain metals, causing several environmental effects. With this study, we aim to characterize the sediments of agricultural areas influenced by agricultural activities in the municipality of Bom Retiro/SC. For significant representation of the area, sediment collections were carried out at 25 points directly influenced by agriculture. The heavy metals cadmium ( $\mathrm{Cd})$, copper $(\mathrm{Cu})$, chromium $(\mathrm{Cr})$, iron $(\mathrm{Fe})$, nickel $(\mathrm{Ni})$, lead $(\mathrm{Pb})$ and zinc $(\mathrm{Zn})$ were used by high atomic absorption spectrometry continuous source resolution (ARFC EAA). Surface sediments exposed to $\mathrm{Cu}, \mathrm{Cr}, \mathrm{Fe}, \mathrm{Ni}, \mathrm{Pb}$ and $\mathrm{Zn}$, but all points are located by CONAMA Resolution n. 454/2012 as level 1.
\end{abstract}

Keywords: Sediment quality; Heavy metals; Agricultural production.

Topic: Química Agrícola e Ambiental

Reviewed anonymously in the process of blind peer.
Received: $10 / 03 / 2020$

Approved: 22/04/2020
Daniely Neckel Rosini (D)

Universidade do Estado de Santa Catarina, Brasil

http://lattes.cnpq.br/1290138248832119

http://orcid.org/0000-0001-9873-6750

danielybio@hotmail.com

Valter Antonio Becegato (D)

Universidade do Estado de Santa Catarina, Brasil

http://lattes.cnpq.br/3196823526572670

http://orcid.org/0000-0002-6850-2846

valter.becegato@udesc.br

\section{Amanda Dalalibera}

Universidade do Estado de Santa Catarina, Brasil

http://lattes.cnpq.br/5815086591868822

amandadalalibera@gmail.com
Pâmela Beccalli Vilela (iD)

Universidade Federal de Minas Gerais, Brasil

http://lattes.cnpq.br/5598572148685197

http://orcid.org/0000-0003-1803-2505

pamelabeccalli@gmail.com

Jordana dos Anjos Xavier

Universidade do Estado de Santa Catarina, Brasil

http://lattes.cnpq.br/5815086591868822

jordanaxavier54@gmail.com

Eduardo Costa Duminelli

Universidade do Estado de Santa Catarina, Brasil

http://lattes.cnpq.br/9872652089189118

eduardoduminelli@gmail.com
Referencing this:

ROSINI, D. N.; BECEGATO, V. A.; DALALIBERA, A.; VILELA, P. B.; XAVIER, J. A.; DUMINELLI, E. C.. Avaliação da qualidade dos sedimentos em áreas agrícolas do município de Bom Retiro/SC. Revista Ibero Americana de Ciências Ambientais, v.11, n.3, p.79-93, 2020. DOI: http://doi.org/10.6008/CBPC2179-6858.2020.003.0008 


\section{INTRODUÇÃO}

Quando a densidade populacional começou a aumentar consideravelmente, a relação entre as espécies também mudou e gerou um crescimento na necessidade da produção de alimentos. A exploração de recursos naturais cresceu para suprir o aumento da demanda por diversos produtos e sustentar um novo estilo de vida (MAHLER, 2012). O Brasil se destaca mundialmente por ser um país com intensa atividade agropecuária (EMBRAPA, 2018). A produção de alimentos em grande escala, que necessita de extensas áreas para cultivo, gera diversos impactos ao ambiente (MAHLER, 2012).

Com o objetivo de aumentar a produção de alimentos, muitos produtos foram desenvolvidos com foco em problemas instantâneos e não em crises planetárias e os países subdesenvolvidos, como o Brasil, acabam utilizando os agroquímicos de forma descontrolada, causando diversos impactos ambientais (GARVEY et al., 2017). Dentre esses impactos está a utilização e contaminação dos recursos naturais (EMBRAPA, 2018). Um desses problemas de contaminação do ambiente em áreas rurais é pelos metais. Alguns metais não são considerados tóxicos dependendo da sua concentração no ambiente (SIQUEIRA-SILVA et al., 2012). Metais como $\mathrm{Cr}$, $\mathrm{Cu}, \mathrm{Ni}, \mathrm{Cr}$, Fe e $\mathrm{Zn}$ são essenciais para os seres vivos, mas outros como $\mathrm{Cd}, \mathrm{Pb}$ e $\mathrm{Hg}$ podem causar sérios danos à saúde humana (FERREIRA et al., 2010). Muitos estão disponíveis naturalmente no solo, mas desde a revolução industrial, a distribuição e disponibilidade têm aumentado significativamente (WAGNER et al., 2014). A aplicação de fertilizantes, agrotóxicos, irrigação e poluição atmosférica são as principais fontes antropogênicas de metais tóxicos em áreas agrícolas. O relevo, o clima, o desenvolvimento econômico, a produção industrial e o estilo de vida da população interferem na disposição dos metais no ambiente (BIGALKE et al., 2017).

Os sedimentos são ambientes de deposição de materiais e compartimentos aquáticos ativos, essenciais para a vida (BEVILACQUA et al., 2009). A alta concentração de metais tóxicos nos sedimentos pode ser uma indicação de poluição antropogênica, ao invés da origem pelo intemperismo (FERREIRA, 2006). Os sedimentos acumulam substâncias tóxicas para os ecossistemas aquáticos, atuando como reservatório de agente contaminante para o ambiente. Muitas substâncias se associam com o material particulado e se incorporam nos sedimentos, agindo como uma fonte de longa duração. Os sedimentos contaminados apresentam risco aos ecossistemas aquáticos e, consequentemente, a todos os ecossistemas adjacentes (QUINÁGLIA, 2006). A análise dos sedimentos pode permitir a detecção de poluentes que podem até estarem ausentes na água, pois estão em constante fluxo sobrejacente com a coluna d'água (JESUS et al., 2004). A alta concentração de metais nos sedimentos pode ser uma indicação de poluição antropogênica, ao invés da origem pelo intemperismo (FERREIRA, 2006).

O objetivo deste estudo foi caracterizar os sedimentos das áreas rurais influenciadas por atividades agropecuárias no município de Bom Retiro/SC e analisar as concentrações dos metais pesados $\mathrm{Cd}, \mathrm{Cr}, \mathrm{Cu}, \mathrm{Fe}$, $\mathrm{Pb}, \mathrm{Ni}$ e Zn, comparando com o que está disposto na legislação brasileira CONAMA n. 454/2012 (CONAMA, 2012). 


\section{METODOLOGIA}

Bom Retiro é um município da região serrana de Santa Catarina, inserido nas sub-bacias Uruguai e Atlântico-Sudeste. Localiza-se a uma latitude 27047'50" sul e a uma longitude 4929'21" oeste, com altitude média de 890 metros. O município tem cerca de nove mil habitantes e a economia se concentra, principalmente, nas atividades agrícolas e pecuárias, entre elas se destaca as culturas de maçã, milho, soja, cebola e tomate (IBGE, 2010). Os pontos de coleta dos sedimentos foram selecionados por estarem localizados nas principais áreas de produção agropecuária de Bom Retiro/SC (Figura 1).

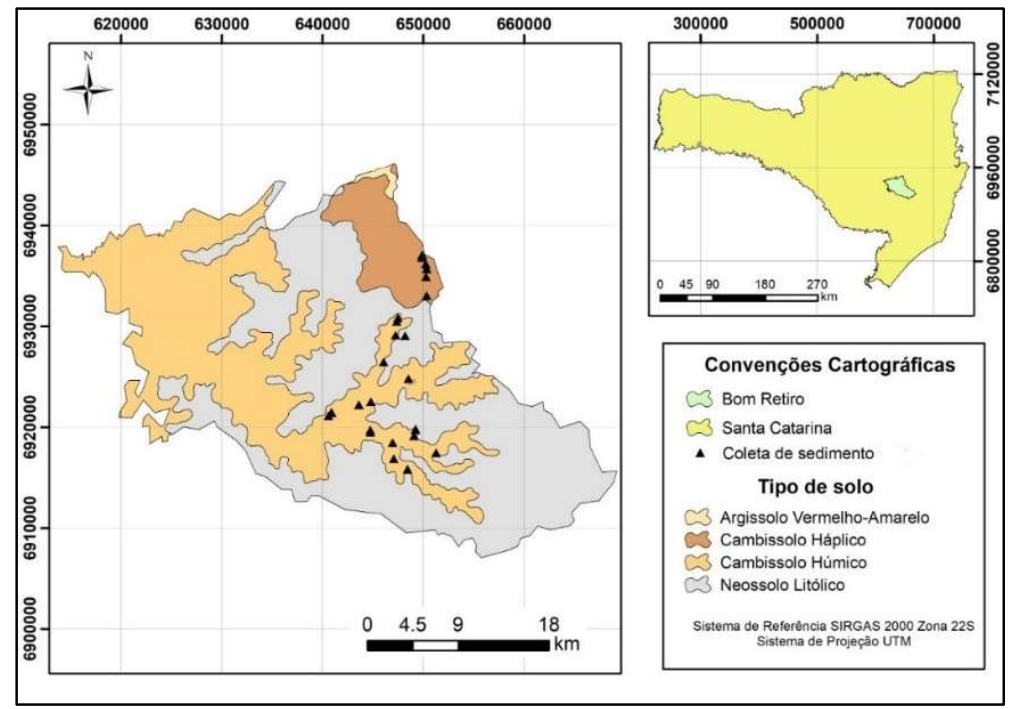

Figura 1: Mapa dos tipos de solo dos pontos de coleta.

Os sedimentos foram coletados nos dias 06 e 07 de dezembro de 2018 em 25 pontos nas margens dos corpos hídricos na profundidade de 0-20 cm (ABNT, 2007). As amostras foram homogeneizadas e retirado uma alíquota para análise, acondicionadas em sacos plásticos devidamente rotulados e encaminhados para o Laboratório de Rotina para Análise de Água e Resíduos - LANAR, do Departamento de Engenharia Ambiental e Sanitária da Universidade do Estado de Santa Catarina - UDESC. Realizou-se análises do potencial hidrogeniônico das amostras, com sonda multiparâmetro portátil HI 98194 marca Hanna (DONAGEMA, 2011). As amostras foram secas em estufa à uma temperatura de $45^{\circ} \mathrm{C}$ por 48 horas (DONAGEMA, 2011), destorroadas e peneiradas em peneira de aço inox de 0,025 mm/ $\mathrm{mm}$ (500 mesh) de malha.

O método 3050 B (APHA, 2012) serviu como base para a digestão das amostras com padrões analíticos da marca Merck para determinação da concentração dos metais Cd (cádmio), Cr (cromo), Cu (cobre), Fe (ferro), Ni (níquel), $\mathrm{Pb}$ (chumbo) e Zn (zinco). A determinação dos metais foi realizada pelo método da chama direta de ar/acetileno, por meio do Espectrofotômetro de Absorção Atômica de Fonte Contínua de Alta Resolução, marca Analytik Jena AG, modelo contrAA 700. Soluções padrões dos metais de interesse foram utilizadas para a calibração do equipamento, com curvas de calibração apropriadas, preparadas por meio de soluções estoques.

Após as análises, os resultados foram comparados com a resolução CONAMA nº 454 (CONAMA, 2012), que estabelece as diretrizes e procedimentos para o gerenciamento de material a ser dragado em 
águas sob jurisdição nacional. Essa resolução estabelece a classificação em dois níveis. No nível 1, limiar abaixo do qual há menor probabilidade de efeitos adversos à biota e no nível 2, há maior probabilidade (Tabela 1).

Tabela 1: Valores orientadores de metais tóxicos para material a ser dragado em água de acordo com a resolução CONAMA n ${ }^{\circ} 454$ de 2012.

\begin{tabular}{|l|l|l|}
\hline Metais $(\mathrm{mg} / \mathrm{kg})$ & Nível 1 & Nível 2 \\
\hline Cádmio & 0,6 & 3,5 \\
\hline Chumbo & 35,0 & 91,3 \\
\hline Cobre & 35,7 & 197,0 \\
\hline Cromo & 37,3 & 90,0 \\
\hline Ferro & $*$ & $*$ \\
\hline Níquel & 18,0 & 35,9 \\
\hline Zinco & 123,0 & 315,0 \\
\hline
\end{tabular}

* Não possui valores definidos na legislação.

A CETESB - Companhia de Tecnologia de Saneamento Ambiental do Estado de São Paulo (2010), possui um guia que permite avaliar contaminantes e define as maiores concentrações permitidas para que não causem efeito adversos nos organismos. O Guia de Critérios para Avaliação da Qualidade dos Sedimentos (Tabela 2) define valores para concentração de metais que classificam a qualidade em ótima, boa, regular, ruim e péssima (CETESB, 2010).

Tabela 2: Classificação dos sedimentos pela CETESB referente à concentração de metais pesados.

\begin{tabular}{|c|c|c|c|c|c|}
\hline & \multicolumn{5}{|c|}{ Parâmetros } \\
\hline & Ótima & Boa & Regular & Ruim & Péssima \\
\hline & \multicolumn{5}{|c|}{ (mg/kg) } \\
\hline Cádmio & $<0,6$ & $0,6-2,1$ & $2,1-3,5$ & $3,5-5,3$ & $>5,3$ \\
\hline Chumbo & $<35,0$ & $35,0-63,2$ & $63,2-91,3$ & $91,3-137,0$ & $>137,0$ \\
\hline Cobre & $<35,7$ & $35,7-116,4$ & $116,4-197,0$ & $197,0-295,5$ & $>295,5$ \\
\hline Cromo & $<37,3$ & $37,3-63,7$ & $63,7-90,0$ & $90,0-135,0$ & $>135,0$ \\
\hline Ferro & $*$ & $*$ & $*$ & $*$ & $*$ \\
\hline Níquel & $<18$ & $18,0-27,0$ & $27,0-36,0$ & $36,0-54,0$ & $>54,0$ \\
\hline Zinco & $<123,0$ & $123,0-219,0$ & $219,0-315,0$ & $315,0-473,0$ & $>473,0$ \\
\hline
\end{tabular}

* Valor não definido. Fonte: CETESB (2010).

\section{RESULTADOS E DISCUSSÃO}

\section{Comportamento Pluviométrico}

As massas de ar que atuam no estado de Santa Catarina são a tropical atlântica, polar atlântica, tropical continental e equatorial continental (OMETTO, 1981). O município de Bom Retiro está inserido em área de interferência do clima mesotérmico úmido, com verão fresco, sem estação seca, com amplitude térmica entre inverno e verão. Nos últimos cinco anos, as menores médias de temperaturas foram observadas no mês de junho e as maiores médias se concentraram entre novembro e dezembro. No ano de realização do estudo, a temperatura mais baixa foi registrada em agosto $\left(-1,94^{\circ} \mathrm{C}\right)$ e nos últimos cinco anos a menor temperatura registrada foi em junho de $2016\left(-3,94^{\circ} \mathrm{C}\right)$.

A precipitação exerce importante função na dispersão e de arraste de material e de sedimentos, por isso tem papel fundamental na análise dos dados (BASSO et al., 2011). A maior temperatura dos últimos cinco anos foi registrada em fevereiro de $2014\left(34,68^{\circ} \mathrm{C}\right)$ e em 2018 a máxima foi observada no mês de dezembro 
$\left(33,62^{\circ} \mathrm{C}\right)$. O valor total da precipitação nos últimos cinco anos teve uma baixa variação, com máxima de 1.667,80 mm em 2016 e mínima de 1.491,00 mm em 2018.

Bom Retiro não tem uma estação seca definida e os maiores índices pluviométricos variam conforme o ano. Vale ressaltar que as chuvas de verão são mais intensas e de curta duração, já as de inverno normalmente são de pouca intensidade e se estendem por vários. Em um estudo sobre a erosividade das chuvas em Lages/SC, Bertol et al. (2002) observaram que a maior parte da chuva erosiva se concentrou nos meses de janeiro, fevereiro e outubro. Durante o verão, a lixiviação de poluentes é maior por causa da intensidade das chuvas e das temperaturas (SILVA et al., 2008).

\section{Potencial Hidrogeniônico}

O potencial hidrogeniônico $(\mathrm{pH})$ é um indicativo de acidez, neutralidade ou alcalinidade, pois indica a concentração de íons hidrogênio $\left(\mathrm{H}^{+}\right)$(LOPES, 2016). O pH é um parâmetro para monitoramento ambiental, pois pode indicar a disponibilidade de metais e a extensão da poluição dos recursos hídricos (OLIVEIRA, 2015). $\mathrm{O}$ pH é influenciado de forma natural ou antropogênica e sofre alterações devido a fotossíntese, adsorção de gases da atmosfera, oxidação da matéria orgânica, dissolução de rochas e composição do solo (PAULA et al., 2010). Os sedimentos tiveram uma variação de pH entre 4,72 a 7,52 e média de 6,36 (Figura 2).

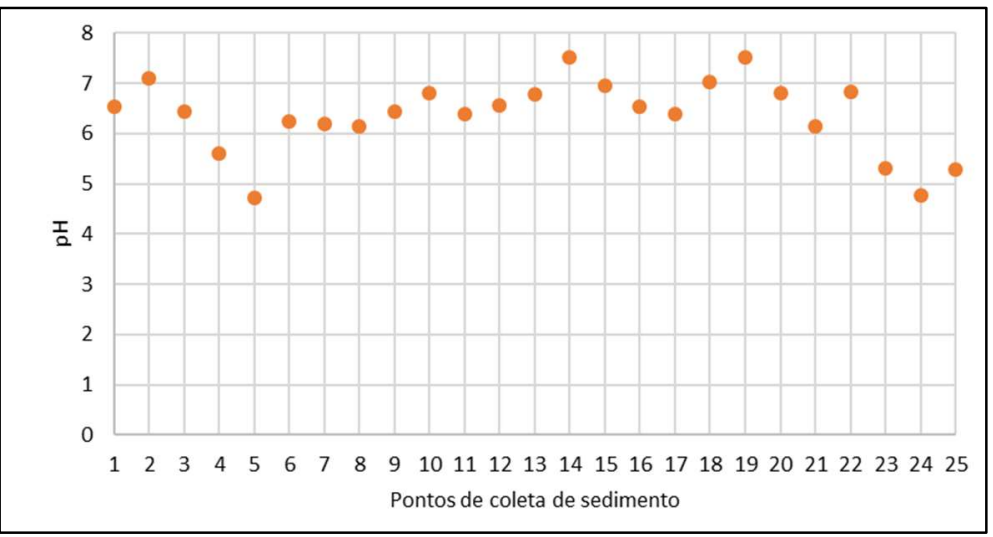

Figura 2: Valores de $\mathrm{pH}$ das amostras analisadas.

\section{Concentração de Metais}

Todas as amostras de água analisadas apresentaram concentrações de $\mathrm{Cd}, \mathrm{Cu}, \mathrm{Ni}, \mathrm{Pb}$ e $\mathrm{Zn}$ abaixo do limite de detecção do aparelho. A concentração de metais pesados na água é maior em períodos chuvosos, porém, no momento da coleta, estava há sete dias sem chover (BATISTA, 2015; LIMA et al., 2015). Os metais podem se acumular em águas quando se tornam mais móveis, pois acontece a lixiviação. A qualidade da água pode ser reduzida e a saúde pública ser posta em risco (ADAMU, 2015). Nas amostras de sedimento, apenas o Cd não foi detectado em nenhuma delas. O Fe foi detectado em todas as amostras, o $\mathrm{Cr}$ em dezessete, o Cu em três, o Zn não foi detectado apenas em uma amostra, o Ni foi encontrado em duas e o Pb em três.

Em todos as amostras de sedimentos foram encontradas concentrações de ferro com limites entre 164,23 e 420,70 mg/kg (Figura 3). O Brasil possui solos e sedimentos com altos teores de óxidos de ferro, os mais comuns são a hematita, característico de solos vermelhos, e a goethita que imprime a cor amarela. Os 
óxidos de ferro adsorvem metais pesados e fixam o fósforo no solo, deixando-o pouco disponível para as plantas. Além disso, os óxidos de ferro afetam a capacidade de troca catiônica dos solos, pois atuam como agentes cimentantes (EMBRAPA, 2019).

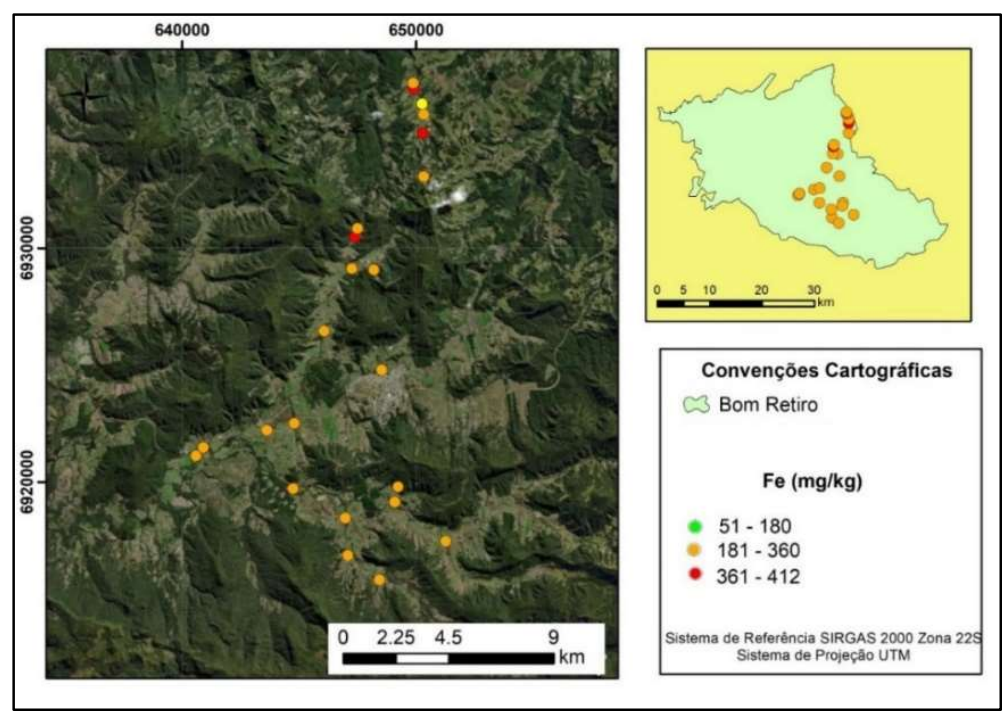

Figura 3: Concentração de ferro nos pontos de coleta de sedimentos.

O intemperismo das rochas e do solo pode fazer com que o ferro seja encontrado na água, principalmente em períodos de altos índices pluviométricos. As reações químicas vão ocasionando transformações que desmancham o arranjo dos minerais. Um exemplo é o processo de oxidação que ocorre quando o ferro ferroso $\left(\mathrm{Fe}^{2+}\right)$ se combina com o oxigênio dissolvido na água para formar o ferro férrico $\left(\mathrm{Fe}^{3+}\right)$ dos óxidos e hidróxidos de ferro. Outro exemplo é o processo de redução, que é o inverso da oxidação (LEPSCH, 2016). É mais comum encontrar ferro nas águas superficiais nas estações chuvosas, por causa do carreamento do solo e erosão nas margens (CETESB, 2009; BATISTA, 2015). Destaca-se que no momento da coleta estava há sete dias sem precipitação.

O uso excessivo de fertilizantes e disposição inadequada de resíduos pode contribuir para o escoamento superficial com água contaminada com ferro e a ausência da mata ciliar contribui para que a água do escoamento superficial atinja o corpo hídrico e os sedimentos (CARVALHO et al., 2017). O sulfato ferroso monohidratado $\left(\mathrm{FeSO}_{4} \cdot \mathrm{H}_{2} \mathrm{O}\right)$, sulfato ferroso heptahidratado $\left(\mathrm{FeSO}_{4} .7 \mathrm{H}_{2} \mathrm{O}\right)$ e o quelato de ferro $\left(\mathrm{C}_{10} \mathrm{H}_{12} \mathrm{~N}_{2} \mathrm{O}_{8} \mathrm{FeNa}_{2}\right)$ são alguns fertilizantes que são utilizados na agricultura e podem contribuir para o aparecimento de ferro nos corpos d'água e nos sedimentos (ANVISA, 2019).

$\mathrm{Na}$ água, o ferro possibilita o surgimento de bactérias ferruginosas nocivas e interfere no sabor, turbidez e descoloração (PHADKE, 2014; BORTOLATTO et al., 2017). Plantas com excesso de ferro, acumulam esse metal e apresentam amarelamento das folhas e escurecimento das raízes. Além disso, o ferro pode atrapalhar na absorção de outros nutrientes como Ca, K, Mg, P e Zn (SIQUEIRA-SILVA et al., 2012). No corpo humano, os níveis de ferro devem ser rigidamente regulados, pois embora haja muitas doenças, como a anemia, vinculadas à deficiência de ferro, o excesso também é muito tóxico ao corpo (TORTORA, 2016; SARKAR, 2018).

O cromo é outro metal que se apresenta no ambiente em diferentes estados de oxidação e podem 
ser essenciais ou tóxicos aos seres vivos. Os compostos de $\mathrm{Cr}$ (II) são iônicos e redutores e se oxidam a Cr (III) quando expostos ao ar. Os compostos de $\mathrm{Cr}$ (III) são os mais estáveis e essenciais, encontrados naturalmente no ambiente. Os compostos de cromo (0) e cromo (VI) possuem facilidade de penetrar nas células, são geralmente produzidos por processos artificiais, através das indústrias de couro e nas ligas metálicas (CAMPOS, 2001; RUPPENTHAL, 2013; QUADRO et al., 2018). Os compostos de cromo (VI) são altamente tóxicos para as plantas e animais e carcinogênicos e mutagênicos para os humanos, irritam os olhos, a pele e as mucosas, sendo a dose letal de apenas algumas gramas (CHEN et al., 2018; CHOUDHARY et al., 2018; PANG et al., 2018; VINCENT, 2018).

Nos sedimentos, a concentração permitida é de até $37,3 \mathrm{mg} / \mathrm{kg}$ de cromo. Porém, apesar de dezessete amostras de sedimentos apresentarem concentrações de cromo, todas estão muito abaixo do limite estabelecido pela legislação (Figura 4) (BRASIL, 2012).

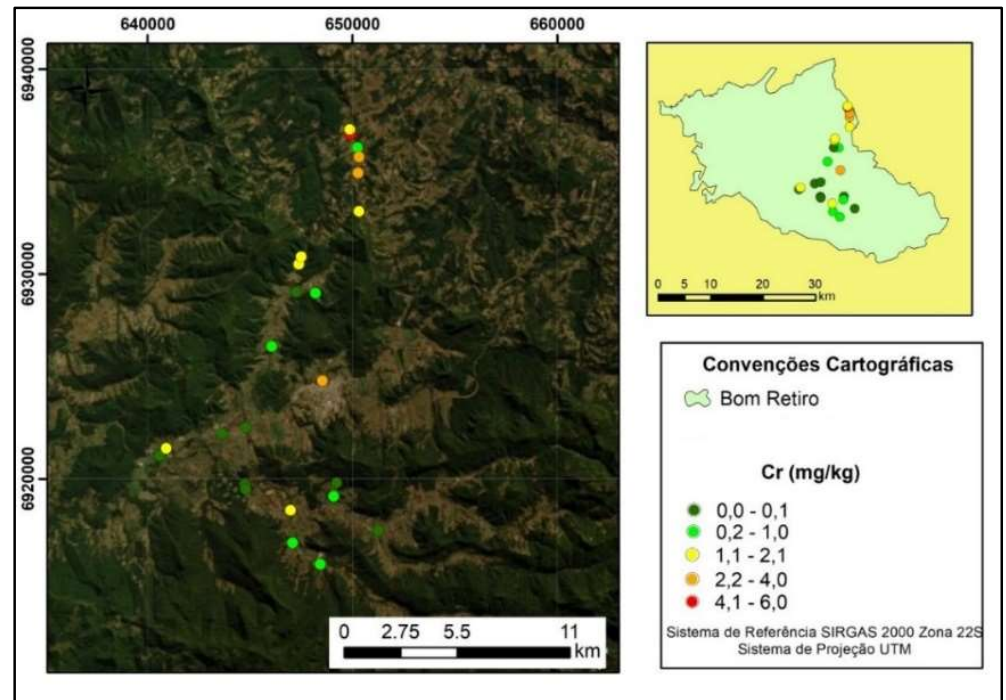

Figura 4: Concentração de cromo nos pontos de coleta de sedimentos.

Os solos presentes na área de estudo apresentam grande parte da CTC ocupada por cátions de $\mathrm{Al}^{3+}$, o que impede que os metais ficam adsorvidos e o risco de lixiviação e contaminação superficial das águas aumenta. Cruz (2012) e Nascimento et al. (2015), constataram aumento na concentração de cromo na água, após o corpo hídrico atravessar o perímetro urbano.

Quando o $\mathrm{Cr}^{6+}$ é reduzido a $\mathrm{Cr}^{3+}$, ele, que era fracamente adsorvido e relativamente móvel no solo, passa a ser pouco móvel, pois é fortemente adsorvido aos coloides do solo (MEURER, 2006). Os fertilizantes fosfatados são a principal fonte de metais, principalmente de $\mathrm{Cd}, \mathrm{Cr}$ e Pb (BIGALKE et al., 2017; SALMAN et al., 2017). A rocha fosfática de onde é extraída esse mineral, é constituída principalmente de apatita e sua fórmula estrutural é $\mathrm{Me}_{10}\left(\mathrm{XO}_{4}\right)_{8} \mathrm{Y}_{2}$ onde Me pode ser $\mathrm{Ca}, \mathrm{Pb}, \mathrm{Zn}, \mathrm{Na}$, Sr ou $\mathrm{Cd}$; $\mathrm{X}$ pode ser $\mathrm{P}, \mathrm{As}, \mathrm{V}, \mathrm{S}, \mathrm{C}$ ou $\mathrm{Si}$ e $\mathrm{Y}$ pode ser $\mathrm{F}, \mathrm{OH}, \mathrm{Cl}$ ou $\mathrm{Br}$ (HARBEN et al., 1990).

Pelo menos dezesseis nutrientes são essenciais ao desenvolvimento das plantas. A eficiência agrícola depende da ação de fertilizantes fosfatados e o consumo de adubos sintéticos para suprir essa necessidade vegetal se torna economicamente viável (CAMPOS, 2001). O calcário (carbonato de cálcio $\left(\mathrm{CaCO}_{3}\right)$ ) é um insumo agrícola muito utilizado para o corrigir o pH do solo. Campos (2001) analisou a composição química 
de calcário dolomítico, muito utilizado na agricultura brasileira e encontrou $3,85 \mathrm{mg} / \mathrm{kg}$ de cromo. Além disso, ele analisou também os fertilizantes sintéticos e encontrou altas concentrações de cromo, como por exemplo o Can-Fal com concentração de 16,57 ppm, o Plant-Fal com 5,32 ppm, o NPK apresentou 508,32 ppm de cromo e o Yoorin Master 979 ppm.

Os compostos de cromo podem causar efeitos cutâneos, com irritação no dorso da mão e nos dedos, podendo causar úlceras; nasais, com irritação e inflamação; pulmonares, com irritação bronquial e alteração da função respiratória; gastrointestinais, através da formação de úlceras; renais e carcinogênicos (BOETCHER, 2008). A principal forma de absorção do cromo é através dos pulmões, mas pode acontecer também através da pele, de onde é levado por meio do sangue a vários órgãos do corpo, onde se concentra principalmente nos rins, fígado, baço e pulmão (RUPPENTHAL, 2013).

$\mathrm{Na}$ agricultura são utilizados muitos produtos que contém cobre com diversos propósitos. O míldio da videira é controlado por meio da aplicação do fungicida Bordeaux, composta por $\mathrm{CuSO}_{4}$ e $\mathrm{Ca}(\mathrm{OH})_{2}$, este

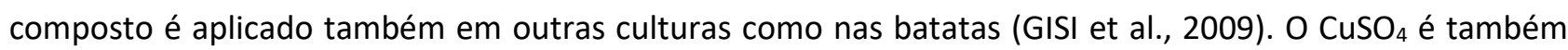
usado em pedilúvios para prevenir e curar a dermatite digital em ovelhas, prática proibida na União Europeia (THOMSEN et al., 2008). Tintas à base de cobre são utilizadas para prevenir o crescimento de microrganismos marinhos em redes na aquicultura (BURRIDGE et al., 2010). O excesso de cobre no corpo humano pode provocar hepatite e distúrbios neurológicos e psiquiátricos (VASCONCELOS, 2012). Mas ele é um elemento essencial, pois auxilia na fixação do ferro na hemoglobina do sangue (KRAVCHENKO-DOVGA, 2018).

Nos sedimentos as concentrações de cobre estão muito abaixo de $35,7 \mathrm{mg} / \mathrm{kg}$, limite máximo de concentração estabelecido para os sedimentos de classe 1 de qualidade ótima (CETESB, 2010; BRASIL, 2012). Em apenas três amostras de sedimentos: na S09 com concentração de 0,059 mg/kg, a S11 0,031 mg/kg e S12 $0,098 \mathrm{mg} / \mathrm{kg}$ (Figura 5).

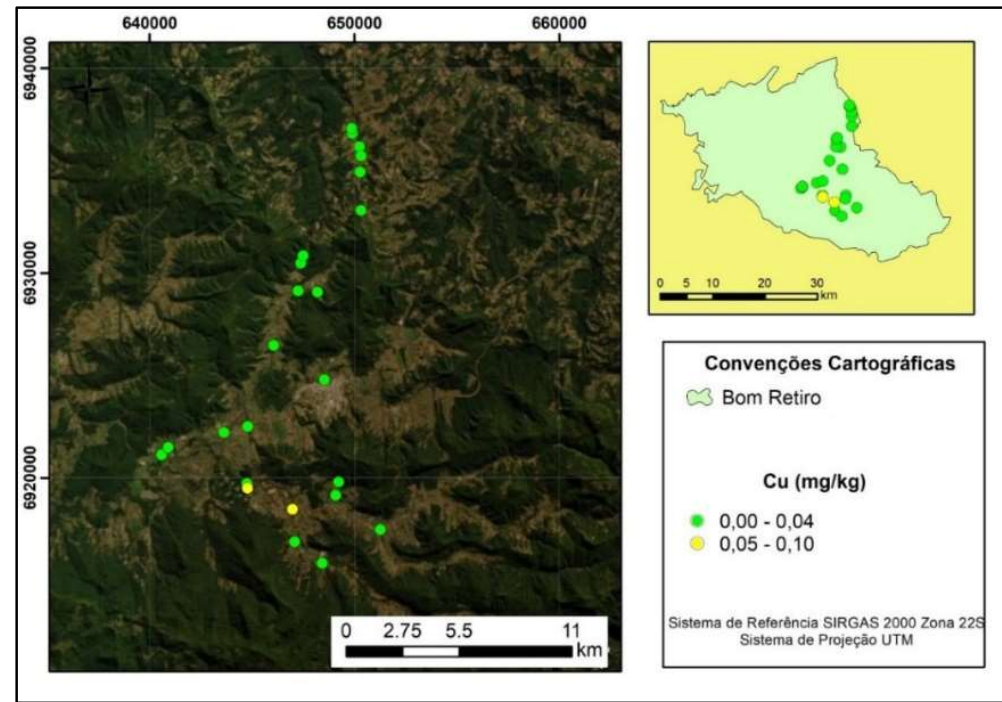

Figura 5: Concentração de cobre nos pontos de coleta de sedimentos.

O zinco é outro metal essencial e compete com o cádmio, um metal tóxico para a saúde humana (CAKMAK et al., 2009). Níveis adequados de zinco, impedem a absorção de cádmio. Nas plantas, o zinco pode auxiliar no aumento da biossíntese de clorofila e carotenoides, fazendo com que o aparato fotossintético da 
planta se torne mais eficaz (ARAVIND et al., 2004). A deficiência de zinco é o fator limitante mais comum nas diversas culturas do mundo (ALLOWAY et al., 2008). Novas tecnologias vêm surgindo para evitar o uso excessivo de fertilizantes e tornar a absorção pelas plantas mais eficaz, evitando a perda de nutrientes e a lixiviação (SINGH et al., 2018). O único ponto de análise de sedimentos que não apresenta concentrações de zinco é um ponto de controle que está localizado em uma área bem preservada, com mata ciliar e pouca interferência antrópica. As concentrações de zinco encontradas oscilaram entre 0,786 e 7,091 mg/kg (Figura $6)$.

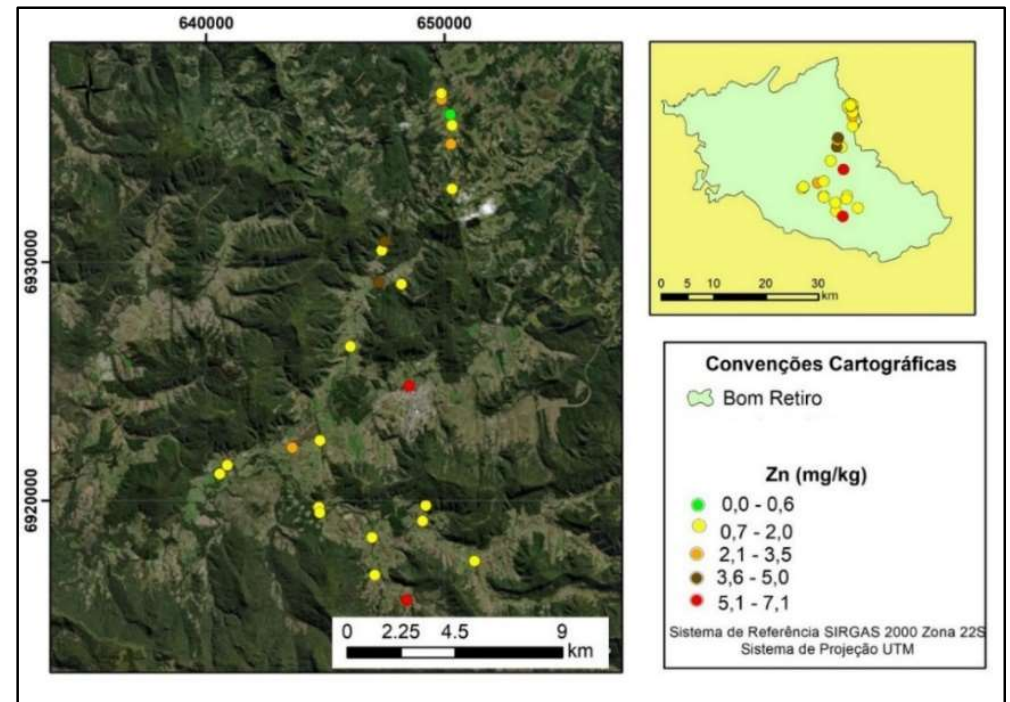

Figura 6: Concentração de zinco nos pontos de coleta de sedimentos.

Os pontos com maiores concentrações de zinco estão associados com a presença da pecuária bovina e suína. Todos os pontos (Figura 6) apresentaram concentração muito abaixo do limite de $123 \mathrm{mg} / \mathrm{kg}$, que é definido pela resolução CONAMA n 454/2012 (CONAMA, 2012) para sedimentos de classe 1 e pela CETESB (2010) para sedimentos considerados de ótima qualidade. Medardus et al. (2014) realizaram um estudo e observaram que a concentração de zinco e cobre nas fezes suínas é maior que nas rações. Em ambientes próximos a áreas de piscicultura, também foram encontradas altas concentrações de zinco e cobre (BURRIDGE et al., 2010). Muitos estudos vêm sendo desenvolvidos, com o objetivo de substituir o zinco inorgânico pelo orgânico na ração animal para ser melhor absorvido pelos indivíduos (JAHANIAN et al., 2015).

O níquel é um elemento presente naturalmente na superfície terrestre (EFSA, 2015). É considerado essencial para as plantas e alguns animais. Está presente na enzima uréase, que atua na hidrólise da ureia e é importante para o metabolismo do nitrogênio (JASMIM et al., 2002). Apenas as amostras dos sedimentos $\mathrm{S} 11(0,303 \mathrm{mg} / \mathrm{kg})$ e $\mathrm{S} 16(0,101 \mathrm{mg} / \mathrm{kg})$ apresentaram concentrações de Ni (Figura 7). Não é vital para as funções humanas, mas alguns compostos de níquel foram classificados como agentes carcinogênicos (VENTURA, 2016; IARC, 2016).

A Agência Internacional de Pesquisa sobre o Câncer classificou os compostos de níquel como carcinogênicos para humanos (Grupo 1), enquanto o Ni-metal é classificado como Grupo 2B, possivelmente carcinogênico (IARC, 1990; IARC, 2012). Inspiração, alimentos e água são as principais fontes de exposição de níquel para a população (GANGULY, 2015). 


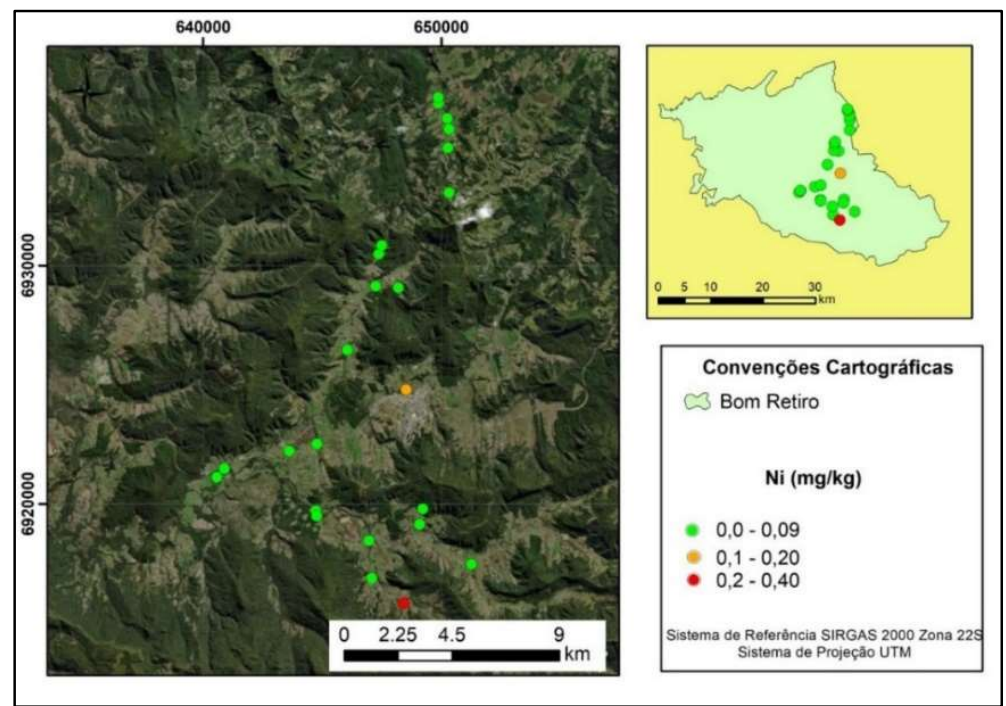

Figura 7: Concentração de níquel nos pontos de coleta de sedimentos.

O chumbo é encontrado em baixas concentrações em rochas calcárias e ultramáficas, e em altas concentrações nas rochas ígneas ácidas e sedimentos argilosos. Está presente naturalmente na rocha-mãe e solos com baixa drenagem possuem capacidade de reter maiores concentrações de metais pesados (FAQUIN, 2005). O chumbo está presente nos aditivos de combustíveis, água potável, efluentes, suplemento de cálcio, pesticidas e fertilizantes (GEBLER, 2007).

Apenas três amostras de sedimentos apresentaram concentrações de chumbo detectáveis pelo aparelho, a S10 com 0,530 mg/kg, S21 com 1,181 mg/kg e a S24 0,151 mg/kg. Conforme a Figura 8, as concentrações detectadas estão muito abaixo do estabelecido pela resolução CONAMA $n^{\circ} 454$ para sedimentos de classe 1 , que é de $35 \mathrm{mg} / \mathrm{kg}$ (CONAMA, 2012).

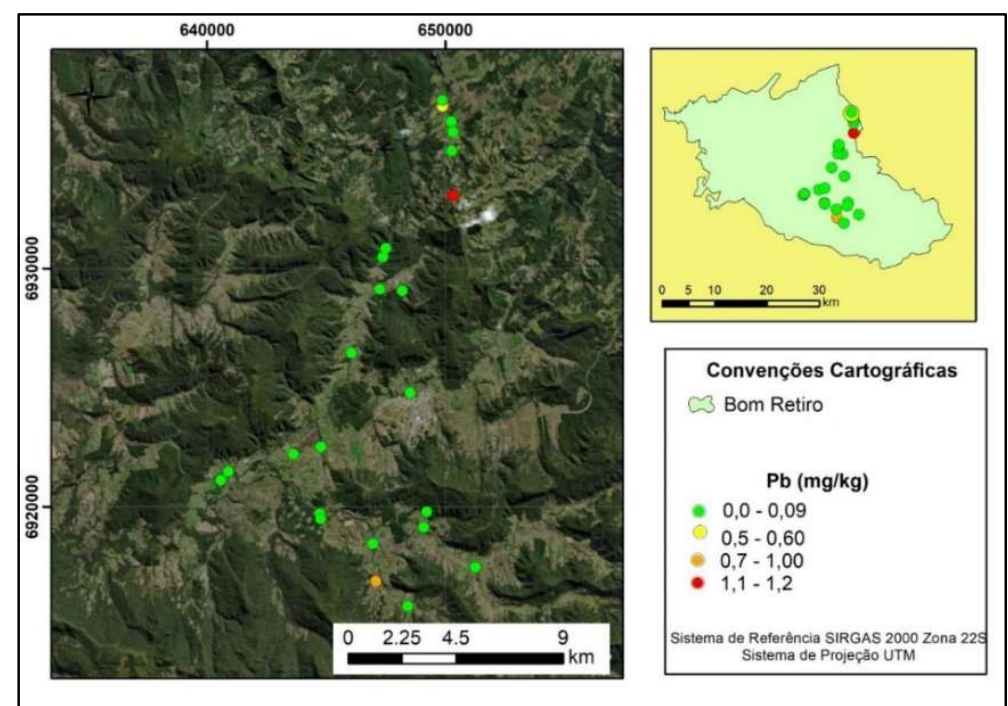

Figura 8: Concentração de chumbo nos pontos de coleta de sedimentos.

O chumbo é um metal que não apresenta efeitos benéficos nutricionais conhecidos para os animais, por isso fica difícil estabelecer limites permitidos para um metal não essencial. Atualmente, é considerado o maior metal poluente ambiental, a contaminação ocorre, na maioria das vezes, por causa da manutenção indevida (XAVIER, 2010). Os metais tóxicos podem ser prejudiciais à saúde humana mesmo em baixas concentrações, podendo se acumular nos tecidos. O chumbo pode causar distúrbios metabólicos, problemas 
cardiovasculares, neurológicos e gastrointestinais, que dependem do nível de exposição (FLORA, 2012; SINGH, 2011).

As concentrações de $\mathrm{Cr}$, Zn, Ni e Pb apresentaram elevada variabilidade, cujo coeficiente de variação foi de $95,55 \% ; 73,80 \% ; 70,68 \%$ e $83,98 \%$ respectivamente. Como o município de Bom Retiro caracteriza-se por diferentes formações geológicas, isso interfere na composição dos diferentes tipos de solos e consequentemente na concentração e disposição dos metais nos sedimentos.

Tabela 3: Estatística descritiva dos metais nos sedimentos.

\begin{tabular}{|l|l|l|l|l|l|l|l|}
\hline & $\mathrm{Fe}$ & $\mathrm{Cr}$ & $\mathrm{Cu}$ & $\mathrm{Zn}$ & $\mathrm{Ni}$ & $\mathrm{Pb}$ & $\mathrm{pH}$ \\
\hline Média & 316,177 & 1,570 & 0,063 & 2,357 & 0,202 & 0,620 & 6,36 \\
\hline Mediana & 321,300 & 1,240 & 0,059 & 1,525 & 0,202 & 0,530 & 6,44 \\
\hline Mínimo & 164,233 & 0,210 & 0,031 & 0,786 & 0,110 & 0,150 & 4,72 \\
\hline Máximo & 420,700 & 5,880 & 0,098 & 7,091 & 0,302 & 1,181 & 7,52 \\
\hline Desvio Padrão & 56,437 & 1,500 & 0,034 & 1,739 & 0,143 & 0,521 & 0,739 \\
\hline Coeficiente de Variação (\%) & 17,85 & 95,55 & 53,93 & 73,80 & 70,68 & 83,98 & 11,63 \\
\hline Assimetria & $-0,782$ & 1,780 & 0,518 & 1,653 & $*$ & 0,758 & $-0,778$ \\
\hline Curtose & 1,341 & 2,378 & $*$ & 2,002 & $*$ & $*$ & 0,338 \\
\hline
\end{tabular}

* Não foi possível estabelecer os valores através de cálculos matemáticos.

A Tabela 4 apresenta a matriz de correlaçãos entre as variáveis analisadas. O período de coleta das amostras de sedimentos se caracterizou por uma época de intensa atividade agrícola, próxima a colheita de cebola e início do plantio de milho, com intensa utilização de agrotóxicos e fertilizantes. Os contaminantes inorgânicos presentes no solo podem ser carreados e afetar as águas superficiais e subterrâneas. A água pode ser o principal transportador de substâncias que se dissolvem nela. A biodisponibilidade e mobilidade dos metais depende do $\mathrm{pH}$, temperatura, potencial redox, CTC, competição com outros metais, ligações aniônicas e a força iônica da solução do solo (PEDROTTI et al., 2012). A sorção é um processo muito importante para a destinação dos poluentes nos sedimentos e na água, pois o soluto adere às superfícies das partículas dos solos (OLIVEIRA et al., 2010).

Tabela 4: Matriz de correlação entre as variáveis relativas aos sedimentos analisadas.

\begin{tabular}{|l|l|l|l|l|l|l|l|}
\hline & $\mathbf{p H}$ & $\mathbf{C u}$ & $\mathbf{C r}$ & $\mathbf{F e}$ & $\mathbf{N i}$ & $\mathbf{P b}$ & $\mathbf{Z n}$ \\
\hline $\mathbf{p H}$ & 1,00 & & & & & & \\
\hline $\mathbf{C u}$ & 0,99 & 1,00 & & & & & \\
\hline $\mathbf{C r}$ & $-0,34$ & 1,00 & 1,00 & & & & \\
\hline $\mathbf{F e}$ & 0,39 & 0,14 & 0,28 & 1,00 & & & \\
\hline $\mathbf{N i}$ & $*$ & $*$ & $-1,00$ & 1,00 & 1,00 & & \\
\hline $\mathbf{P b}$ & 0,54 & $*$ & $-0,68$ & $-1,00$ & $*$ & 1,00 & \\
\hline $\mathbf{Z n}$ & 0,08 & $-0,73$ & 0,19 & $-0,04$ & 1,00 & $-0,74$ & 1,00 \\
\hline
\end{tabular}

* Não foi possível estabelecer os valores por meio de cálculos matemáticos.

A toxicidade dos metais é controlada por propriedades físicas e químicas. A distribuição dos metais no solo sofre influência da mineralogia, textura, classificação e pH (ZHANG et al., 2007). Alguns estudos demonstraram o aumento da mobilidade de metais devido à baixa do pH (HU, 2006). Essa mobilidade pode depender do tempo de contato (PLASSARD, 2000). O pH interfere nos processos de especiação química (VASCONCELLOS, 2015). Muitas reações podem ser controladas através do pH e ORP, pois diversas substâncias, como os metais, podem ter sua toxicidade e mobilidade alteradas por causa deles, principalmente em sistemas aquáticos naturais que se tem seu equilíbrio termodinâmico alterado 
constantemente (JARDIM, 2014). A agricultura contemporânea não tem como fonte de contaminação ambiental apenas o uso dos agrotóxicos, mas também através da adubação sintética. Muitos metais são encontrados nos agroquímicos (CAMPOS, 2001).

\section{CONCLUSÕES}

A área de estudo está inserida em uma região com intensa atividade agropecuária. $\mathrm{Na}$ análise dos sedimentos, nenhum ponto apresentou concentrações de $\mathrm{Cd}$, dois continham $\mathrm{Ni}$, três $\mathrm{Pb}$, três $\mathrm{Cu}$, dezessete $\mathrm{Cr}$, vinte e quatro tinham $\mathrm{Zn}$ e todos continham $\mathrm{Fe}$, provavelmente devido a composição das rochas e dos solos. Como nenhum ponto de coleta de sedimentos apresentou concentrações acima do que está disposto na legislação, possivelmente os metais utilizados na agricultura lixiviam para o corpo hídrico, por onde podem espalhar contaminação pelo ambiente.

O período de coletadas amostras de sedimentos caracterizou-se pelo intenso uso de agroquímicos, devido aos diferentes estágios de desenvolvimento vegetativo das culturas. A declividade dos terrenos onde acontece o cultivo agrícola pode contribuir para a lixiviação dos metais. A agricultura gera impactos ao meio, que podem ser mitigados através de um maior controle dos produtos utilizados.

\section{REFERÊNCIAS}

ABNT. Associação Brasileira de Normas Técnicas. Norma Brasileira n. 15.492: Sondagem de reconhecimento para fins de qualidade ambiental: Procedimento. Rio de Janeiro: ABNT, 2007.

ADAMU, C. I.; NGANJE, T. N.; EDET, A.. Heavy metal contamination and health risk assessment associated with abandoned barite mines in Cross River State, southeastern Nigeria. Environmental Nanotechnology, Monitoring \& Management, v.3, p.10-21, 2015.

ALLOWAY, B. J.; GRAHAM, R.; STACEY, S.. Micronutrient deficiencies in Australian field crops: In Micronutrient deficiencies in global crop production. Springer Netherlands, 2008.

ALMODOVAR, M. L. N.. A origem natural da poluição por cromo no Aquífero Adamantina, município de Urânia SP. Tese (Doutorado em Geociências) - Universidade de São Paulo, São Paulo, 2000.

ANVISA. Agência Nacional de Vigilância Sanitária. Regularização de Produtos: Agrotóxicos. ANVISA, 2019.

APHA. American Public Health Association. Standard methods for the examination of water and wastewater. 22 ed. Washington: APHA, 2012.

ARAVIND, P.; PRASAD, M. N. V.. Zinc protects chloroplasts and associated photochemical functions in cadmium exposed Ceratophyllum demersum L., a fresh water macrophyte. Plant Sci., v.166, p.1321-1327, 2004.

BATISTA, M. A. R. S.. Avaliação da qualidade da água e sedimento de microbacias utilizadas para abastecimento público da região metropolitana de Goiânia. Dissertação
(Mestrado em Engenharia do Meio Ambiente) Universidade Federal de Goiás, Goiânia, 2015.

BERTOL, I.. Erosividade das chuvas e sua distribuição entre 1989 e 1998 no município de Lages (SC). Revista Brasileira de Ciência do Solo, v.26, n.2, 2002.

BERTOLO, R. A.; MARCOLAN, L. N. O.; BOUROTTE, C. L. M. Water-Rock Interaction and the Hydrogeochemistry of Chromium in Groundwater from Multilevels Monitoring Wells in Urania, SP, Brazil. 2009.

BEVILACQUA, J. E.; SILVA, I. S.; LICHTIG, J.; MASINI, J. C.. Extração seletiva de metais pesados em sedimentos de fundo do rio Tietê, São Paulo. Química Nova, v.32, n.1, p.2633, 2009.

BIGALKE, M.; ULRICH, A.; REHMUS, A.; KELLER, A. Accumulation of cadmium and uranium in arable soils in Switzerland. Environ. Pollut., v.221, p.85-93, 2017.

BOETCHER, M. L.. Cromo: aspectos toxicológicos e ocupacionais. Monografia (Bacharelado em Ciências Farmacêuticas) - Universidade Feevale, Novo Hamburgo, 2008

BORTOLATTO, R.; LENHARD, D. C.; GENENA, A. K.. Evaluation of a natural coagulant in the polishing treatment of swine slaughterhouse wastewater. Desalination and Water Treatment, v.97, p.126-132, 2017.

CONAMA. Conselho Nacional do Meio Ambiente. Resolução n. 454, de 01 de dezembro de 2012. Estabelece as diretrizes gerais e os procedimentos referenciais para o gerenciamento do material a ser dragado em águas sob jurisdição nacional. Brasília: DOU, 2012. 
BURRIDGE, L.; WEIS, J. S.; CABELLO, F.; PIZARRO, J.; BOSTICK $K$.. Chemical use in salmon aquaculture: a review of current practices and possible environmental effects. Aquaculture, v.306, n.14, p.7-23, 2010.

CAKMAK, I.; KALAYCI, M.; KAYA, Y.; TORUN, A. A.; AYDIN, N.; WANG, Y.. Biofortification and localization of zinc in wheat grain. Journal of Agricultural \& Food Chemistry, v.58, p.9092-9102, 2010.

CAMPOS, V.. Comportamento químico de arsênio, fósforo e metais pesados (cromo, cobre, chumbo e mercúrio) em solos expostos a cultivares frutíferos, Município de Jundiaí, São Paulo. Tese (Doutorado) - Universidade de São Paulo, São Paulo, 2001.

CARVALHO, S. S.; CRUZ, M. A. S.; AMORIM, J. R. A.; ARAGÃO, R. D.. Presença de cádmio e ferro em amostras da água da sub-bacia hidrográfica do rio Siriri, Estado de Sergipe. In: EMBRAPA TABULEIROS COSTEIROS-ARTIGO EM ANAIS DE CONGRESSO; SIMPÓSIO BRASILEIRO DE RECURSOS HÍDRICOS, 22. Anais. Porto Alegre: Associação Brasileira de Recursos Hídricos, 2017.

CETESB. Companhia Ambiental do Estado de São Paulo. Índices de qualidade das águas, critérios de avaliação da qualidade dos sedimentos e indicador de controle de fontes: Apêndice B. Série Relatórios. São Paulo: CETESB, 2010.

CHARLET, L.; MANCEAU, A. A.. X-Ray absorption spectroscopic study of the sorption of $\mathrm{Cr}$ (III) at the oxide water interface II. Adsorption, coprecipitation and surface precipitation on hydrous ferric oxide. Journal of Colloid and Interface Science, v.148, p.443-458, 1992.

CHEN, H.; GUO, Z.; ZHOU, Y.;LI, D.; MU, L.; KLERKS, P.L.; LUO Y.; XIE, L.. Accumulation, depuration dynamics and effects of dissolved hexavalent chromium in juvenile Japanese medaka. Ecotox Environ Safe, v.148, p.254-260, 2018.

CHOUDHARY, B.; PAUL, D.. Isotherms, kinetics and thermodynamics of hexavalent chromium removal using biochar. J. Environ. Chem. Eng., v.6, p.2335-2343, 2018.

CRUZ, M. A. S.. Avaliação da geoquímica dos sedimentos superficiais das nascentes do Rio Subaé/BA. Dissertação (Mestrado em Modelagem em Ciências da Terra e Ambiente) - Universidade Estadual de Feira de Santana, Feira de Santana, 2012.

DONAGEMA, G. K.. Manual de métodos de análise de solos. Rio de Janeiro: Embrapa Solos, 2011.

EFSA. Panel on Contaminants in the Food Chain. Scientific Opinion on the risks to public health related to the presence of nickel in food and drinking water. EFSA Journal, 2015.

EMBRAPA. Empresa Brasileira de Pesquisa Agropecuária. Visão 2030: o futuro da agricultura brasileira. Brasília: EMBRAPA, 2018.

EMBRAPA. Empresa Brasileira de Pesquisa Agropecuária. Teor de óxidos de Ferro. Brasília: EMBRAPA, 2018.
FAQUIN, V.. Nutrição mineral de plantas. Lavras: UFLA, 2005.

FERREIRA, J. G.. Avaliação de parâmetros ambientais em aterros sanitários: estudo de caso. Taubaté, 2006.

FERREIRA, A. P.; HORTA, M. A. P.; CUNHA, C. L. N.. Avaliação das concentrações de metais pesados no sedimento, na água e nos órgãos de Nycticorax nycticorax (Garça-da-noite) na Baía de Sepetiba, RJ, Brasil. 2010. Journal of Integrated Coastal Zone Management, v.10, n.2, p.229-241, 2010.

FLORA, G.; GUPTA, D.; TIWARI, A.. Toxicity of lead: a review with recent updates. Interdisciplinary toxicology, v.5, n.2, p.47-58, 2012.

GANGULY, R.; PIERCE, G. N.. The toxicity of dietary trans fats. Food Chem Toxicol., v.78, p.170-176, 2015.

GARVEY, B.; TYFIELD, D.; MELLO, L.. Meet the New Boss: Same as the old boss? Tchnology, toil and tension in the agrofuel frontier. New Technology, Work and Employment, v.30, n.2, p.79-94, 2017

GEBLER, L.; PALHARES, J. C. P.. Gestão ambiental na agropecuária. Bento Gonçalves: Embrapa Uva e Vinho, 2007.

GISI, U.; SIEROTZKI, H.; COOK, A.; MCCAFFERY, A.

Mechanisms influencing the evolution of resistance to Qo inhibitor fungicides. Pest management science, v.58, n.9, p.859-867, 2009

HARBEN, P. W.; BATES, R. L.. Industrial minerals geology and worls deposits. In: Phosphate rock. London: Metal Bullerin, 1990.

IARC. International Agency for Research on Cancer. Monographs on the Evaluation of Carcinogenic Risks to Humans: A Review of Human Carcinogens. Lyon: International Agency for Research on Cancer, 2016.

IARC. International Agency for Research on Cancer. Chromium, nickel and welding. IARC monographs, 1990.

IARC. International Agency for Research on Cancer. Arsenic, metals, fibres, and dusts. IARC monographs, 2012.

IBGE. Instituto Brasileiro de Geografia e Estatística. IBGE Cidades. Rio de Janeiro: IBGE, 2010.

JAHANIAN, R.; RASOULI, E.. Effects of dietary substitution of zinc-methionine for inorganic zinc sources on growth performance, tissue zinc accumulation and some blood parameters in broiler chicks. J. Anim. Physiol., v.99, p.50-58, 2015.

JARDIM, W. F.. Medição e interpretação de valores do potencial redox (Eh) em matrizes ambientais. Quim. Nova, v.37, n.7, p.1233-1235, 2014.

JASMIM, J. M.; MONNERAT, P. H.; ROSA, R. C. C.. Efeito da omissão de $\mathrm{N}, \mathrm{Ni}$, Co e $\mathrm{S}$ sobre os teores de $\mathrm{N}$ e $\mathrm{S}$ em feijoeiro. Revista Brasileira de Ciência do Solo, v.26, p.967975, 2002.

JESUS, H. C.; COSTA E. A.; MENDONÇA, A. S. F.; ZANDONADE, E.. Distribuição de metais pesados em sedimentos do 
sistema estuário da Ilha de Vitória/ES. Revista Química Nova, Vol. 27, No. 3, 378-386, 2004.

KRAVCHENKO-DOVGA, Y. V.; KARPOVSKY, V. I.; DANCHUK, O. $\mathrm{V}$.. The role of the main characteristics of cortical processes in the regulation of exchanges of Cuprum. Scientific Messenger of LNU of Veterinary Medicine and Biotechnologies, v.20, n.83, p.295-298, 2018.

LEPSCH, I. F.. Formação e conservação dos solos. Oficina de textos, 2016.

LIMA, D. P.; SANTOS, S.; SILVA, R. A.; YOSHIOKA, E. T. O; BEZERRA, R. M.. Contaminação por metais pesados em peixes e água da bacia do rio Cassiporé, Estado do Amapá, Brasil. Acta Amazonica, v.45, n.4, p.405-414, 2015.

LOSI, M. E.; AMRHEIN, C.; FRANKENBERGER JUNIOR, W. T. Environmental biochemistry of chromium. Reviews of Environmental Contamination and Toxicology, v.136, p.91121, 1994

MAHLER, C. F.. Lixo urbano: o que você precisa saber sobre o assunto. Rio de Janeiro: FAPERJ, 2012.

MEDARDUS, J. J.; MOLLA, B. Z.; NICOL, M.; MORROW, W. M.; RAJALA-SCHULTZ, P. J.; KAZWALA, R.; GEBREYES, W. A.. Infeed use of heavy metal micronutrients in US swine production systems and its role in persistence of multidrugresistant salmonellae. Appl. Environ. Microb., v.80, p.23172325, 2014.

MEURER, E. J.. Fundamentos de química do solo. 3 ed. Porto Alegre: Evangraf, 2006

NASCIMENTO, B. L. M.. Comportamento e avaliação de metais potencialmente tóxicos (Cu (II), $\mathrm{Cr}$ (III), $\mathrm{Pb}$ (II) e Fe (III)) em águas superficiais dos Riachos Capivara e Bacuri Imperatriz/MA, Brasil. Engenharia Sanitária e Ambiental, v.20, n.3, p.369-378, 2015.

OLIVEIRA, L. F. C.. Isotermas de sorção de metais pesados em solos do cerrado de Goiás. Revista Brasileira de Engenharia Agrícola e Ambiental, v. 14, n. 7, p. 776-782, 2010.

OMETTO, J. C.. Bioclimatologia vegetal. São Paulo: Agronômica Ceres, 1981.

PANG, L.; HU, J.; ZHANG, M.; YANG, C.; WU, G.. An efficient and reusable quaternary ammonium fabric adsorbent prepared by radiation grafting for removal of $\mathrm{Cr}(\mathrm{VI})$ from wastewater. Environ. Sci. Pollut Res., v.25, p.11045-11053, 2018.

PEDROTTI, A.; MELLO, A. V.. Modelagem da produção de sedimentos na sub-bacia hidrográfica do Riacho Jacaré/SE. 2012.

PHADKE, A.. Iron Removal Using Electro-Coagulation Followed by Floating Bead Bed Filtration (MSc thesis). Louisiana State University and Agriculturaland Mechanical College, 2014

PLASSARD, F.; WINIARSKI, T.; PETIT-RAMEL, M.. Retention and distribution of three heavy metals in a carbonated soil: comparison between batch and unsaturated column studies.
Journal of Contaminant Hydrology, v.42, p.99-111, 2000.

QUADRO, M. S.; ANDREAZZA, R.; TEDESCO, M. J.; GIANELO, C.; BARCELOS, A. A.; BORTOLON, L.. Chromium contents linked to iron oxide at areas with tannery sludge disposal. Engenharia Sanitária e Ambiental, v.23, n.1, p.63-67, 2018.

QUINÁGLIA, G. A.. Caracterização dos níveis basais de concentração de metais de sedimentos do sistema estuarino da baixada santista. São Paulo: Universidade de São Paulo, 2006

RAI, D.; EARY, L. E.; ZACHARA, J. M.. Environmental chemistry of chromium. The Science of the Total Environment, v.86, p.15-23, 1989

RIBEIRO, T. G.. Estudo Da Qualidade Das Águas Por Meio Da Correlação De Parâmetros Físico-Químicos, Bacia Hidrográfica Do Ribeirão Anicuns. Geochimica Brasiliensis, v.30, n.1, p.84-94, 2017.

RICHARD, F. C.; BOURG, A. C. M.. Aqueous geochemistry of chromium: a review. Water Research, v.25, p.807-816, 1991.

RUPPENTHAL, J. E.. Toxicologia. Santa Maria: Colégio Técnico Industrial da Universidade Federal de Santa Maria, 2013.

SALMAN, S. A.; ELNAZER, A. A.; NAZER, H. A. E.. Integrated mass balance of some heavy metals fluxes in Yaakob village, south Sohag, Egypt. Int. J. Environ. Sci. Technol., v.14, n.5, p.1011-1018, 2017.

SARKAR, J.; POTDAR, A. A.; SAIDEL, G. M.. Whole-body iron transport and metabolism: Mechanistic, multi-scale model to improve treatment of anemia in chronic kidney disease. PLoS computational biology, v.14, n.4, 2018.

SINGH, A.; AFZAL, S.; SINGH, T.; HUSSAIN, I.. Zinc oxide nanoparticles: a review of their biological synthesis, antimicrobial activity, uptake, translocation and biotransformation in plants. Journal of Materials Science, v.53, n.1, p.185-201, 2018.

SILVA, A. E. P.. Influência da precipitação na qualidade da água do Rio Purus. Acta Amazonica, v.38, n.4, p.733-742, 2008.

SIQUEIRA-SILVA, A. I.; A. I.; SILVA, L. C.; AZEVEDO, A. A.; OLIVA, M. A.. Iron plaque formation and morphoanatomy of roots from species of resting subjected to excess iron. Ecotoxicology and Environmental Safety, v.78, p.265-275, 2012.

THOMSEN, W. J.; GROTTICK, A. J.; MENZAGHI, F.; REYESSALDANA, H.; ESPITA, S.; YUSKIN, D.. Lorcaserin, a novel selective human 5-hydroxytryptamine2C agonist: in vitro and in vivo pharmacological characterization. J. Pharmacol Exp. Ther., v.325, p.577-587, 2008.

TORTORA, G. J.; DERRICKSON, B.. Corpo Humano: Fundamentos de Anatomia e Fisiologia. Porto Alegre: Artmed, 2016.

VASCONCELLOS, G. R.. Avaliação das condições de oxirredução em sistema alagado construído de escoamento horizontal subsuperficial. Dissertação (Mestrado em 
Saneamento, Meio Ambiente e Recursos Hídricos) -

Universidade Federal de Minas Gerais, Belo Horizonte, 2015.

VASCONCELOS, P. D. S.. Monitoramento da Água de Diálise: Um Estudo de Caso em uma Clínica do Município de Recife. Dissertação (Especialização em Gestão de Sistemas e Serviços de Saúde) - Fundação Oswaldo Cruz, 2012.

VENTURA, M.. Ocorrência de níquel em alimentos consumidos em Portugal: resultados preliminares do projeto-piloto. Lisboa: Total Diet Study, 2016.

VINCENT, J.. The nutritional biochemistry of chromium. Elsevier, 2018.
WAGNER, A.; KAUPENJOHANN, M.. Suitability of biochars (pyro- and hydrochars) for metal immobilization on former sewage-field soils. Eur. J. Soil Sci., v.65, p.139-148, 2014.

XAVIER, J. M.. Quantificação de metais essenciais e tóxicos em dejetos depositados naturalmente nas pastagens oriundos da pecuária leiteira familiar do alto da bacia do Rio Paraná em Goiás, Brasil Central, Brasil. 2010.

ZHANG, M.; ZHENG, S.. Competitive Adsorption of $\mathrm{Cd}, \mathrm{Cu}, \mathrm{Hg}$ and $\mathrm{Pb}$ by Agricultural Soils of the Changjiang and Zhujiang Deltas in China. Journal of Zhejiang University Science A, Hangzhou, v.8, p.1808-1815, 2007.

A CBPC - Companhia Brasileira de Produção Científica (CNPJ: 11.221.422/0001-03) detém os direitos materiais desta publicação. Os direitos referem-se à publicação do trabalho em qualquer parte do mundo, incluindo os direitos às renovações, expansões e disseminações da contribuição, bem como outros direitos subsidiários. Todos os trabalhos publicados eletronicamente poderão posteriormente ser publicados em coletâneas impressas sob coordenação da Sustenere Publishing, da Companhia Brasileira de Produção Científica e seus parceiros autorizados. Os (as) autores (as) preservam os direitos autorais, mas não têm permissão para a publicação da contribuição em outro meio, impresso ou digital, em português ou em tradução. 